Ann. Inst. Statist. Math.

Vol. 46, No. 1, 57-66 (1994)

\title{
THE SINGLY TRUNCATED NORMAL DISTRIBUTION: A NON-STEEP EXPONENTIAL FAMILY
}

\author{
JOAN DEL CASTILLO \\ Department of Mathematics, Universitat Autònoma de Barcelona, \\ 08193 Bellaterra, Barcelona, Spain
}

(Received September 17, 1992; revised January 21, 1993)

\begin{abstract}
This paper is concerned with the maximum likelihood estimation problem for the singly truncated normal family of distributions. Necessary and suficient conditions, in terms of the coefficient of variation, are provided in order to obtain a solution to the likelihood equations. Furthermore, the maximum likelihood estimator is obtained as a limit case when the likelihood equation has no solution.
\end{abstract}

Key words and phrases: Truncated normal distribution, likelihood equation, exponential families.

\section{Introduction}

Truncated samples of normal distribution arise, in practice, with various types of experimental data in which recorded measurements are available over only a partial range of the variable. The maximum likelihood estimation for singly truncated and doubly truncated normal distribution was considered by Cohen (1950, 1991). Numerical solutions to the estimators of the mean and variance for singly truncated samples were computed with an auxiliar function which is tabulated in Cohen (1961). However, the condition in which there is a solution have not been determined analytically.

Barndorff-Nielsen (1978) considered the description of the maximum likelihood estimator for the doubly truncated normal family of distributions, from the point of view of exponential families. The doubly truncated normal family is an example of a regular exponential family. In this paper we use the theory of convex duality applied to exponential families by Barndorff-Nielsen as a way to clarify the estimation problems related to the maximum likelihood estimation for the singly truncated normal family, see also Rockafellar (1970).

The singly truncated normal family of distributions is a natural and practical example of a non-regular and non-steep exponential family; for more information see Efron (1978) and Letac and Mora (1990). The complete description of the mean domain of this family is given in Theorem 4.2, that shows likelihood equation, 
solved numerically by Cohen, has only one solution if and only if the sample coefficient of variation is less than 1 . If the sample coefficient of variation is greater than 1, which happens with positive probability, then the maximum likelihood estimator yields a distribution of the usual one-parameter exponential family. In the paper we also give numerical functions defined in MATHEMATICA which provided the solutions to the likelihood equations, and a short table, computed with these functions, which can be used to obtain a first approximation to the solutions. Without loss of generality, only distributions left truncated by zero are considered. The usual changes $x-x_{l}$ for a random variate $x$, left truncated by $x_{l}$, or $x_{r}-x$ for a random variate $x$, right truncated by $x_{r}$, can be used to reduce the problem to the previous situation.

\section{Exponential families}

Let $\mu$ be a $\sigma$-finite positive measure on a $\sigma$-algebra of subsets of a measurable space $\mathcal{X}$ and let $T$ be a statistic on $\mathcal{X}$ over $\mathbb{R}^{k}$. Let $\mu_{T}$ be the marginal distribution of $T$ and let us consider its Laplace transform

$$
L_{T}(\theta)=\int_{\mathcal{X}} e^{\theta^{\dagger} T} d \mu=\int_{\mathbb{R}^{k}} e^{\theta^{\dagger} t} d \mu_{T}, \quad \theta \in \mathbb{R}^{k} .
$$

We denote the existence domain of $L_{T}$ by $D=\left\{\theta: L_{T}(\theta)<\infty\right\}$ (which is convex, by Hölder's inequality) and the interior of $D$ by $\Theta=\operatorname{int}(D)$, which we will assume is not empty.

Let us consider the family of distributions

$$
\mathcal{P}_{D}=\left\{p(x ; \theta) d \mu=e^{\theta^{\dagger} T(x)-K_{T}(\theta)} d \mu: \theta \in D\right\}
$$

where $K_{T}(\theta)=\ln L_{T}(\theta)$ is a strictly convex function on $\Theta$, from Hölder's inequality, and real analytic on $\Theta$. The family $\mathcal{P}_{D}$ is called a full exponential family and $D$ is called its natural parameter space. $\mathcal{P}_{D}$ is said to be regular if $D=\Theta$; if not then we will also consider $\mathcal{P}_{\Theta}=\{p(x ; \theta) d \mu: \theta \in \Theta\}$. Let $S$ be the support of the measure $\mu_{T}$ and let $C$ be the closed convex hull of $S$. Let us assume that $S$ is not included in an affine submanifold of $\mathbb{R}^{k}$. Then $T$ is a minimal sufficient statistic for family $\mathcal{P}_{D}$.

For $\theta \in \Theta$, the mean value and the covariance matrix of $T$ can be obtained by differentiation

$$
\begin{aligned}
& E_{\theta} T=\frac{\partial K_{T}}{\partial \theta}, \\
& V_{\theta} T=\frac{\partial^{2} K_{T}}{\partial \theta \partial \theta^{\dagger}} .
\end{aligned}
$$

Since $K_{T}$ is strictly convex on $\Theta$, the map $\tau(\theta)=E_{\theta} T$ is one-to-one. Denote by $M_{T}$ the image of $\Theta$ by $\tau, M_{T}$ is refered to as the domain of means of $\mathcal{P}_{\Theta}$. Clearly, $M_{T}$ is included in the interior of $C$. If $M_{T}=\operatorname{int}(C)$ then family $\mathcal{P}_{D}$ is said to be steep. In most of the cases the full exponential families are regular or 
steep, but not always, as we shall see in Section 4 (see also Efron (1978) and Letac and Mora (1990)).

Let $x=\left(x_{1}, x_{2}, \ldots, x_{n}\right)^{\dagger}$ be a random sample of a distribution $p(x ; \theta) d \mu \in \mathcal{P}_{D}$. The log-likelihood function for the family $\mathcal{P}_{D}$ is

$$
l(\theta ; x)=n\left(\theta^{\dagger} t-K_{T}(\theta)\right), \quad \theta \in D
$$

where $t=\sum_{i=1}^{n} T\left(x_{i}\right) / n$. Then, the likelihood equations for a full exponential family are

$$
\tau(\theta)=E_{\theta} T=t, \quad \theta \in \Theta .
$$

Note that the likelihood estimation method for $\theta \in \Theta$ corresponds to the method of moments for the statistic $T$. The function $\theta^{\dagger} t-K_{T}(\theta)$ is the Legendre transform of $K_{T}(\theta)$ and the conjugate of the convex function $K_{T}(\theta)$

$$
\hat{l}(t)=\sup _{\theta \in D}\left\{\theta^{\dagger} t-K(\theta)\right\}
$$

is called the sup-log-likelihood function (see Barndorff-Nielsen (1978)).

The effective domain of $\hat{l}(t)$ is the set $\operatorname{dom}(\hat{l})=\{t: \hat{l}(t)<+\infty\}$ which corresponds to the set of points for which maximum likelihood estimate exist. The following result gives us a good approximation of dom $(\hat{l})$; its proof can be found in Barndorff-Nielsen ((1978), p. 140).

THEOREM 2.1. With the notations given above, we have

$$
\operatorname{int}(C) \subset \operatorname{dom}(\hat{l}) \subset C,
$$

in particular, for a random sample $x=\left(x_{1}, x_{2}, \ldots, x_{n}\right)^{\dagger}$, if $t=\sum T\left(x_{i}\right) / n$ is in $\operatorname{int}(C)$ then there is a maximum of the likelihood function $l(\theta, x)$ on $D$.

\section{The truncated normal distribution}

Let us consider the full exponential family $\mathcal{P}_{D}$ given by $\mathcal{X}=\mathbb{R}_{+}, d \mu(x)=d x$ the Lebesgue measure on $\mathbb{R}_{+}$and $T(x)=\left(x,-x^{2}\right)$. The Laplace transform of $\mu_{T}$ is

$$
L_{T}\left(\theta_{1}, \theta_{2}\right)=\int_{0}^{\infty} e^{\theta_{1} x-\theta_{2} x^{2}} d x, \quad\left(\theta_{1}, \theta_{2}\right) \in \mathbb{R}^{2}
$$

straighforward integration shows that $L_{T}\left(\theta_{1}, \theta_{2}\right)$ is finite on $D=\Theta \cup \Theta_{0}$, where $\Theta=\operatorname{int}(D)=\mathbb{R} \times \mathbb{R}_{+}$and $\Theta_{0}=\{(\theta, 0): \theta<0\}$, and we have

$$
\begin{aligned}
& L_{T}\left(\theta_{1}, \theta_{2}\right)=\sqrt{\pi / \theta_{2}} \phi\left(\theta_{1} / \sqrt{2 \theta_{2}}\right) e^{\theta_{1}^{2} / 4 \theta_{2}}, \quad\left(\theta_{1}, \theta_{2}\right) \in \Theta, \\
& L_{T}(\theta, 0)=-\frac{1}{\theta}, \quad(\theta, 0) \in \Theta_{0}
\end{aligned}
$$


where $\phi(x)$ is the distribution function of the standard normal.

Let us consider for $\left(\theta_{1}, \theta_{2}\right) \in \Theta$, the parametrization $\theta_{1}=\mu / \sigma^{2}$ and $\theta_{2}=$ $1 / 2 \sigma^{2}$, then the probability density functions of distributions of $\mathcal{P}_{\Theta}$ are

$$
p\left(x ; \theta_{1}, \theta_{2}\right)=(\sqrt{2 \pi} \sigma \phi(\mu / \sigma))^{-1} \exp \left(-(x-\mu)^{2} / 2 \sigma^{2}\right), \quad 0<x<\infty,
$$

which shows that $\mathcal{P}_{\Theta}$ is the family of singly truncated normal distributions (see Cohen (1991)). On the other hand, we can consider the family $\mathcal{P}_{\Theta_{0}}$ of the distributions of $\mathcal{P}_{D}$ with parameters $(\theta, 0) \in \Theta_{0}$. Clearly $\mathcal{P}_{\Theta_{0}}$ is the usual one-parameter exponential family of distributions.

$$
p(x ; \theta, 0)=-\theta e^{\theta x}, \quad \theta<0, \quad 0<x<\infty .
$$

Now, we shall study the regularity of $K_{T}\left(\theta_{1}, \theta_{2}\right)=\ln L_{T}\left(\theta_{1}, \theta_{2}\right)$ on $D$. To this end we introduce some notation. Let

$$
K(x)=\ln \int_{0}^{\infty} e^{x t-t^{2} / 4} d t, \quad x \in \mathbb{R}
$$

then $K(x)$ is a convex function, since it is the logarithm of a Laplace transform, and $K_{T}\left(\theta_{1}, \theta_{2}\right)=-\ln \left(2 \sqrt{\theta_{2}}\right)+K\left(\theta_{1} / 2 \sqrt{\theta_{2}}\right)$. Let $k(x)$ be the derivative of $K(x)$. The following expressions are available for $k(x)$.

$$
k(x)=2 x+e^{-x^{2}} / E(x)=2 x+e^{-x^{2}}(\sqrt{\pi} \phi(\sqrt{2} x))^{-1}=2 x+\sqrt{2} / R(-\sqrt{2} x)
$$

where $E(x)=\sqrt{\pi} \phi(\sqrt{2} x)$ is the error function and $R(x)=\sqrt{2 \pi} \phi(-x) e^{x^{2} / 2}$ is the Mills' ratio.

Note that for $\left(\theta_{1}, \theta_{2}\right) \in \Theta$ the map $\tau\left(\theta_{1}, \theta_{2}\right)=E_{\left(\theta_{1}, \theta_{2}\right)}(T), \tau=\left(\tau_{1}, \tau_{2}\right)^{\dagger}$, may be expressed as

$$
\begin{aligned}
& \tau_{1}\left(\theta_{1}, \theta_{2}\right)=\frac{\partial K_{T}}{\partial \theta_{1}}=\theta_{1}^{-1} x k(x) \\
& \tau_{2}\left(\theta_{1}, \theta_{2}\right)=\frac{\partial K_{T}}{\partial \theta_{2}}=-2 \theta_{1}^{-2}\left(x^{2}+x^{3} k(x)\right)
\end{aligned}
$$

where $x=\theta_{1} / 2 \sqrt{\theta_{2}}$.

Proposition 3.1. (a) The functions $L_{T}\left(\theta_{1}, \theta_{2}\right)$ and $K_{T}\left(\theta_{1}, \theta_{2}\right)$ are $\mathcal{C}^{1}$ functions on $D=\Theta \cup \Theta_{0}$, with

$$
\frac{\partial K_{T}}{\partial \theta_{1}}(\theta, 0)=\theta^{-1}, \quad \frac{\partial K_{T}}{\partial \theta_{2}}(\theta, 0)=-2 \theta^{-2} .
$$

(b) The probability density function $p\left(x ; \theta_{1}, \theta_{2}\right),\left(\theta_{1}, \theta_{2}\right) \in \Theta$, converges to $p(x ; \theta, 0),(\theta, 0) \in \Theta_{0}$, in $L^{1}\left(\mathbb{R}_{+} d x\right)$ as $\left(\theta_{1}, \theta_{2}\right)$ tends to $(\theta, 0)$.

Proof. $L_{T}\left(\theta_{1}, \theta_{2}\right)$ and $K_{T}\left(\theta_{1}, \theta_{2}\right)$ be real analytic on $\Theta$, then let us assume $\left(\theta_{1}, \theta_{2}\right)$ is in a neighborhood of $(\theta, 0), \theta<0$. If $\left(\theta_{1}, \theta_{2}\right)$ tends to $(\theta, 0)$, then $\exp \left(\theta_{1} x-\theta_{2} x^{2}\right)$ converges to $\exp (\theta x)$ for all $x$ in $\mathbb{R}_{+}$and for some $\epsilon>0, \theta+\epsilon<0$,

$$
\left|\exp \left(\theta_{1} x-\theta_{2} x^{2}\right)\right| \leq \exp ((\theta+\epsilon) x) .
$$


From the Lebesgue bounded convergence theorem it follows that $L_{T}\left(\theta_{1}, \theta_{2}\right)$ and $K_{T}\left(\theta_{1}, \theta_{2}\right)$ are continuous in $D$. The continuity of $L_{T}\left(\theta_{1}, \theta_{2}\right)$ and the inequality (3.6) also prove (b), by Lebesgue bounded convergence theorem.

When $x$ tends to $-\infty$ we can show the following limits hold

$$
\lim _{x \rightarrow-\infty} x k(x)=-1, \quad \lim _{x \rightarrow-\infty}\left(x^{3} k(x)+x^{2}\right)=1 .
$$

If $\left(\theta_{1}, \theta_{2}\right)$ tends to $(\theta, 0)$ then $x=\theta_{1} / 2 \sqrt{\theta_{2}}$ tends to $-\infty$ and, from (3.5), we deduce that $\left(\tau_{1}, \tau_{2}\right)$ tends to $\left(\theta^{-1},-2 \theta^{-2}\right)$. This show that mapping $\left(\tau_{1}, \tau_{2}\right)$, defined on $\Theta$, can be extended continuously to $\Theta_{0}$ and $L_{T}\left(\theta_{1}, \theta_{2}\right)$ and $K_{T}\left(\theta_{1}, \theta_{2}\right)$ are in $\mathcal{C}^{1}(D)$.

From this proposition it now follows that family $\mathcal{P}_{D}$ is not a steep family, that is, the maximum likelihood estimate is not always a solution to likelihood equations (see Barndorff-Nielsen (1978)), but we shall show this directly in the next section.

\section{Maximum likelihood estimates}

Let $x=\left(x_{1}, x_{2}, \ldots, x_{n}\right)$, be a random sample of a distribution of $\mathcal{P}_{D}$. Let $t_{1}=\sum_{i=1}^{n} x_{i} / n, t_{2}=-\sum x_{i}^{2} / n$. Then $\left(t_{1}, t_{2}\right) \in C$ where

$$
C=\left\{(x, y) \in \mathbb{R}^{2}: x \geq 0, y \leq-x^{2}\right\}
$$

because $C$ is convex, and $\left(t_{1}, t_{2}\right) \in \operatorname{int}(C)$ with probability one, because $n>$ 1. If $\left(t_{1}, t_{2}\right) \in \operatorname{int}(C)$ we know, from Theorem 2.1, that there is a maximum likelihood estimate $\left(\hat{\theta}_{1}, \hat{\theta}_{2}\right)$ in $D$. We note that if $\left(\hat{\theta}_{1}, \hat{\theta}_{2}\right)$ is a solution of the likelihood equations (2.3), where $\tau=\left(\tau_{1}, \tau_{2}\right)^{\dagger}$ is given in $(3.5)$, then $\left(\hat{\theta}_{1}, \hat{\theta}_{2}\right) \in \Theta$ and the maximum likelihood estimate is a distribution of the singly truncated normal family, $\mathcal{P}_{\Theta}$. In this case, we can express the likelihood equations in terms of parameters $\mu=\theta_{1} / 2 \theta_{2}, \sigma^{2}=1 / 2 \theta_{2}$ by

$$
\begin{aligned}
& E\left(t_{1}\right)=\frac{\sigma}{\sqrt{2}} k(\mu / \sqrt{2} \sigma)=t_{1}, \\
& E\left(t_{2}\right)=-\sigma^{2}-\frac{\mu \sigma}{\sqrt{2}} k(\mu / \sqrt{2} \sigma)=t_{2} .
\end{aligned}
$$

In particular we see that likelihood equations correspond to estimations by the method of moments.

If $\left(t_{1}, t_{2}\right)$ is not included in the domain of means of $\mathcal{P}_{\Theta}, \tau(\Theta)$, then $\left(\hat{\theta}_{1}, \hat{\theta}_{2}\right) \in \Theta_{0}$ and the maximum likelihood estimate is a distribution of the usual one-parameter exponential family.

Proposition 4.1. Let $s^{2}=-\left(t_{2}+t_{1}^{2}\right)$, the standard deviation, and $c=s / t_{1}$, the sample coefficient of variation. The likelihood equations (4.2) are equivalent to the equation

$$
\left(1+c^{2}\right) k(x)^{2}-2 x k(x)-2 x=0, \quad x \in \mathbb{R}
$$


with $\hat{\sigma}=\sqrt{2} t_{1} / k(x)$ and $\hat{\mu}=2 t_{1} x / k(x)$.

ProOF. If $x=\mu / \sqrt{2} \sigma$ then, from (4.2), we have

$$
\left(1+c^{2}\right) k(x)=-\frac{t_{2}}{t_{1}^{2}} k(x)=\frac{2}{k(x)}+2 x
$$

and this equation is equivalent to (4.3). Also from (4.2), we have $\sigma=\sqrt{2} t_{1} / k(x)$ and then $\mu=\sqrt{2} \sigma x=2 t_{1} x / k(x)$.

THEOREM 4.2. The domain of means of the singly truncated normal family of distribution $\mathcal{P}_{\Theta}$, is the set of points

$$
M=\left\{(x, y) \in \mathbb{R}^{2}: x>0,-2 x^{2}<y<-x^{2}\right\} .
$$

Equivalently, the likelihood equations (4.2) have a solution if and only if the sample coefficient of variation is less than 1 and then the solution is unique.

Proof. In the same notation of Proposition 4.1, the equation (4.3) is equivalent to

$$
k(x)-f_{c}(x)=0, \quad x \in \mathbb{R}
$$

where $f_{c}(x)=\left(x+\left(x^{2}+2\left(1+c^{2}\right)\right)^{1 / 2}\right) /\left(1+c^{2}\right)$. Functions $k(x)$ and $f_{c}(x)$ are positive and infinitely often differentiable on $\mathbb{R}$. If $x$ tends to $+\infty, f_{c}(x)$ has the asymptote $y=2 x /\left(1+c^{2}\right)<2 x$ then $k(x)$ is bigger than $f_{c}(x)$ if $x$ is big enough. When $x$ tends to $-\infty$ the following limit hold

$$
\lim _{x \rightarrow-\infty} x f_{c}(x)=-1, \quad \lim _{x \rightarrow-\infty}\left(x^{3} f_{c}(x)+x^{2}\right)=\left(1+c^{2}\right) / 2
$$

then, from (3.7), when $x$ tends to $-\infty$, we have the following asymptotic developments

$$
\begin{aligned}
& k(x)=-\frac{1}{x}+\frac{1}{x^{3}}+o\left(1 / x^{3}\right), \\
& f_{c}(x)=-\frac{1}{x}+\frac{1+c^{2}}{2 x^{3}}+o\left(1 / x^{3}\right)
\end{aligned}
$$

then if $-2 t_{1}^{2}<t_{2}<-t_{1}^{2}$ or, equivalently, $1+c^{2}=-t_{2} / t_{1}^{2}<2, k(x)$ is smaller than $f_{c}(x)$ and this shows that there is a solution to equation (4.3). The solution, provided there is one, is always unique, because $\tau$ is $1-1$.

Conversely, if $\tau(\Theta)$ is not included in $M$ then there exist $\left(\theta_{1}, \theta_{2}\right) \in \Theta$ such that $\tau\left(\theta_{1}, \theta_{2}\right)=\left(\tau_{1}, \tau_{2}\right) \in M_{0}=\left\{-2 x^{2}=y, x>0\right\}$, because $\tau(\Theta)$ is a connected set. From the Theorem of inverse mapping $\tau$ is $1-1$ between neighbourhoods of $\left(\theta_{1}, \theta_{2}\right)$ and $\left(\tau_{1}, \tau_{2}\right)$. From Proposition 3.1 we find that $\tau\left(\Theta_{0}\right)=M_{0}$. Then, from continuity of $\tau_{1}$ it follows that $\tau$ is not $1-1$ on $\Theta$ and this is a contradiction.

From the last Theorem we have the following result, which is also proved in Barlow-Proschan (1965). 
COROLLARY 4.3. Let $x$ be a random variate with a singly truncated normal distribution, given by (3.3). Let $E_{\theta} x=\tau_{1}$ be its mean value and $V_{\theta} x=-\left(\tau_{2}+\tau_{1}^{2}\right)$ the variance then we have, for all $\theta \in \Theta\left(V_{\theta} x\right)^{1 / 2}<E_{\theta} x$.

Theorem 4.2 gives a description of maximum likelihood estimates when the sample coefficient of variation, $c$, is less than 1 , but $c$ may be bigger than 1 with positive probability. In fact, Proposition 3.1 show that if $\left(\theta_{1}, \theta_{2}\right)$ is close to $(\theta, 0)$, the probability density functions that correspond to these parameters are close to $L^{1}\left(\mathbb{R}_{+}\right)$, then the probability $P\left\{c \geq 1 ; \theta_{1}, \theta_{2}\right\}$ is close to $P\{c \geq 1 ; \theta\}$, for the one-parameter exponential family (3.4), with parameter $\theta$. In the next section we give some estimates of this probability.

COROLLARY 4.4. Let $s^{2}=-\left(t_{2}+t_{1}^{2}\right)$, the standard deviation, and $c=s / t_{1}$, the sample coefficient of variation. If $c \geq 1$ then the maximum likelihood estimate for the full exponential family $\mathcal{P}_{D}$ is a point $(\hat{\theta}, 0)$ of the limiting case $\mathcal{P}_{\Theta_{0}}$. The point $\hat{\theta}$ is then the solution of the likelihood equation for the one-parameter exponential family (3.4).

Proof. If $c \geq 1$ it follows, from Theorem 4.2, that $\left(t_{1}, t_{2}\right) \notin M=\tau(\Theta)$, then the maximum likelihood estimate is in $\Theta_{0}$. From (3.2) we see that the loglikelihood function, when $(\theta, 0) \in \Theta_{0}$, may be reduced to $n\left(\theta t_{1}+\ln (-\theta)\right)=0$ and this is the log-likelihood equation for family (3.4).

\section{Some numerical approaches}

The probability that the sample coefficient of variation is bigger than 1 for the one-parameter exponential family may be estimated roughly from the following result.

Proposition 5.1. Let $x_{1}, x_{2}, \ldots, x_{n}$ be a random sample of a one-parameter exponential family with distribution $p(x ; \theta)=\theta e^{-\theta x}, \theta>0, x \in \mathbb{R}_{+}$. If $t_{1}=$ $(1 / n) \Sigma x_{i}, t_{2}=-(1 / n) \Sigma x_{i}^{2}$ then $\sqrt{n}\left(-\theta^{2}\left(t_{2}+2 t_{1}^{2}\right) / 2+1 / n\right)$ converge in distribution to the standard normal distribution, $N(0,1)$.

Proof. We can use the asymptotic distribution of the empirical moments $\left(t_{1},-t_{2}\right)$, then if we transform it by a $\mathcal{C}^{1}$ function, we obtain

$$
E_{\theta}\left(-\left(t_{2}+2 t_{1}^{2}\right)\right)=-\frac{2}{n \theta^{2}} \quad \text { and } \quad V_{\theta}\left(-\left(t_{2}+2 t_{1}^{2}\right)\right)=\frac{4}{n \theta^{4}}+o(1 / n)
$$

and the result follows.

Now, if $s^{2}=-\left(t_{2}+t_{1}^{2}\right)$ and $c=s / t_{1}$ we can estimate the probability

$$
P\{c \geq 1\}=P\left\{t_{1} \leq-\left(t_{2}+t_{1}^{2}\right)\right\}=P\left\{\sqrt{n}\left(-\theta^{2}\left(t_{2}+2 t_{1}^{2}\right) / 2+1 / n\right) \geq 1 / \sqrt{n}\right\}
$$

by $1-\phi(1 / \sqrt{n})$, for large $n$. 
For small samples we have estimated the probability $P\{c \geq 1\}$, for the oneparameter exponential family by simulation. We used 5,000 random samples of size $n$, for $n=10,20,30,50$ from a generator of MATHEMATICA and we obtained the following table.

Table 1. Approximate values for $P\{c \geq 1\}$.

\begin{tabular}{cccccc}
\hline$n$ & 10 & 20 & 30 & 40 & 50 \\
\hline$P\{c \geq 1\} \approx$ & 0.254 & 0.319 & 0.347 & 0.352 & 0.364 \\
$1-\phi(1 / \sqrt{n})$ & 0.376 & 0.412 & 0.478 & 0.437 & 0.444 \\
\hline
\end{tabular}

When the sample coefficient of variation, $c$, is less than 1 , the solution to the likelihood equation (4.2) can be obtained, of course, using the auxiliary function $\theta(\gamma)$, where $\gamma=c^{2}$, which is tabulated by Cohen (1961). Then, the solution is

$$
\hat{\mu}=\bar{x}\left(1-\theta\left(c^{2}\right)\right), \quad \hat{\sigma}=s^{2}+\theta\left(c^{2}\right) \bar{x}^{2}
$$

where $\bar{x}$ and $s$ are the sample mean and the standard deviation. The relationship between $\theta\left(c^{2}\right)$ and the solution $x(c)$ to the equation (4.3), or equivalently (4.4), is given by

$$
2 x(c)^{2}=\frac{c^{2}+\theta\left(c^{2}\right)}{\left(1-\theta\left(c^{2}\right)\right)^{2}} .
$$

The solution to (4.2) can also be obtained from the equation (4.4) and the functions $\mathrm{mu}[m, s]$ and sigma $[m, s]$ defined in MATHEMATICA by

$$
\begin{aligned}
& k\left[x_{-}\right]:=2 x+(2 / \operatorname{Sqrt}[\mathrm{Pi}]) E\left(-x^{\wedge} 2\right) / \text { Erf }[- \text { infinity, } x] \\
& f\left[x_{-}, c_{-}\right]:=\left(x+\operatorname{Sqrt}\left[x^{\wedge} 2+2\left(1+c^{\wedge} 2\right)\right] /\left(1+c^{\wedge} 2\right)\right. \\
& x\left[c_{-}\right]:=x / . \text { FindRoor }[k[x]-f[x, c],\{x,\{-10,10\}\}, \text { AccuracyGoal } \rightarrow 10] \\
& \operatorname{sigma}\left[m_{-}, s_{-}\right]:=\operatorname{Sqrt}[2] m / k[x[s / m]] / / \mathrm{N} \\
& \operatorname{mu}\left[m_{-}, s_{-}\right]:=2 m x[s / m] / k[x[s / m]] / / \mathrm{N}
\end{aligned}
$$

where $m=\bar{x}=t_{1}$ and $s=-\left(t_{2}+t_{1}^{2}\right)^{1 / 2}$.

In order to facilitate a first estimation of truncated normal distribution we give Table 2, which expresses the solution $x(c)$, to the equation (4.4) when the coefficient of variation $c$ ranges between 0.30 and 0.99 . Table 2 has been computed using the last function $x[c]$. From Table 2 we can find the maximum likelihood estimation for parameters $\mu, \sigma$ of the truncated normal family by

$$
\hat{\sigma}=\sqrt{2} \bar{x} / f_{c}(x(c)), \quad \hat{\mu}=\sqrt{2} \hat{\sigma} x(c)
$$

where $f_{c}(x)=\left(x+\left(x^{2}+2\left(1+c^{2}\right)\right)^{1 / 2}\right) /\left(1+c^{2}\right)$, because $x(c)$ is a solution of equation (4.4). 


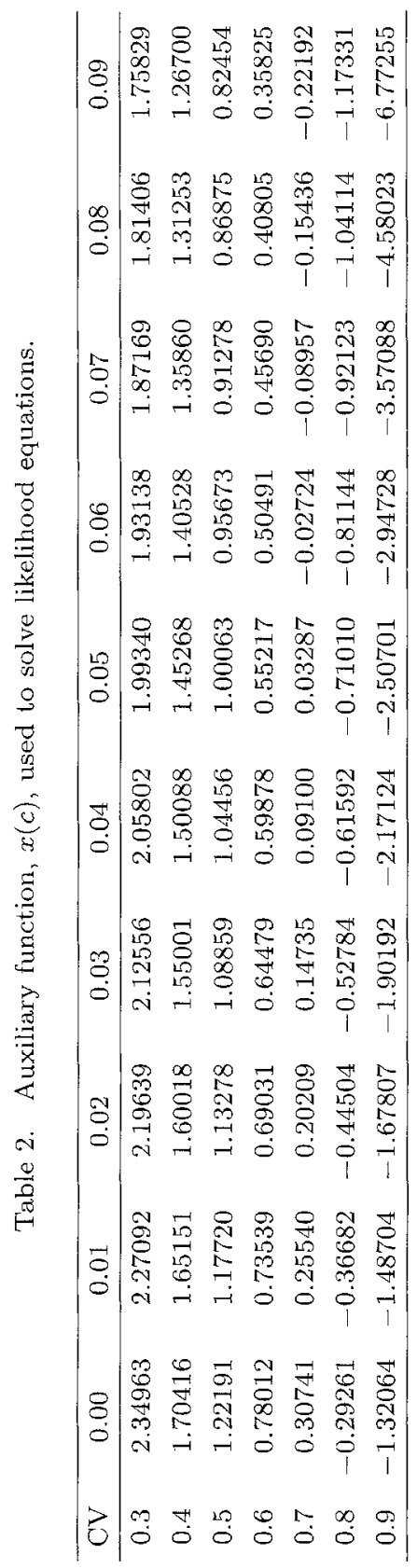




\section{REFERENCES}

Barlow, R. and Proschan, F. (1975). Statistical Theory of Reliability and Life Testing, Holt Rinehart and Winston, New York.

Barndorff-Nielsen, O. (1978). Information and Exponential Families, Wiley, Norwich.

Cohen, A. C. (1950). Estimating the mean and variance of normal populations from singly truncated and doubly truncated samples, Ann. Math. Statist., 21, 557-569.

Cohen, A. C. (1961). Tables for maximum likelihood estimates: singly truncated and singly censored samples, Technometrics, 3, 535-541.

Cohen, A. C. (1991). Truncated and Censored Samples, Marcel Dekker, New York.

Efron, B. (1978). The geometry of exponential families, Ann. Statist., 6, 362-376.

Letac, G. and Mora, M. (1990). Natural real exponential families with cubic variance functions, Ann. Statist., 18, 1-37.

Rockafellar, R. (1970). Convex Analysis, Princeton University Press, New Jersey. 